\title{
The prevalence of root dilacerations in a Turkish population
}

\author{
Ozkan Miloglu ${ }^{1}$, Fatih Cakici ${ }^{2}$, Fatma Caglayan ${ }^{3}$, Ahmet-Berhan Yilmaz ${ }^{4}$, Fatma Demirkaya ${ }^{5}$
}

${ }^{1}$ Assistant Professor, Department of Oral Diagnosis and Radiology, Faculty of Dentistry, Ataturk University, Erzurum, Turkey

${ }^{2}$ Research Assistant, Department of Endodontic, Faculty of Dentistry, Ataturk University, Erzurum, Turkey

${ }^{3}$ Assistant Professor, Department of Oral Diagnosis and Radiology, Faculty of Dentistry, Ataturk University, Erzurum, Turkey

${ }^{4}$ Professor, Department of Oral Diagnosis and Radiology, Faculty of Dentistry, Ataturk University, Erzurum, Turkey

${ }^{5}$ Assistant Professor, Department of Analytical Chemistry, Faculty of Pharmacy, Ataturk University, Erzurum, Turkey

Correspondence:

Department of Oral Diagnosis and Radiology

Faculty of Dentistry, Ataturk University

25240, Erzurum, Turkey

omiloglu@hotmail.com

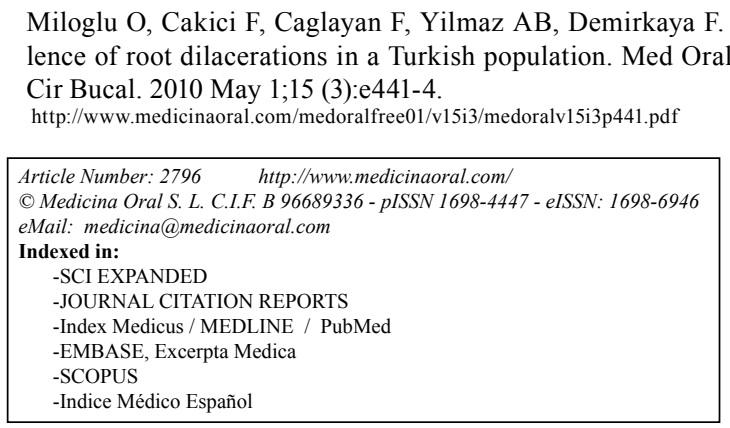

\begin{abstract}
Objectives: The aim of this study was to determine the prevalence of root dilaceration in a Turkish dental patient population with respect to sexes and dental localization in relation between sex and this anomaly.

Study Design: A retrospective study was performed using periapical radiography of 2,124 patients ranging in age from 15 to 65. All data (age, sex and systemic disease or syndrome) was obtained from the files. These patients were analyzed for root dilaceration. Descriptive characteristics of sexes, jaws, and dental localization were recorded. The Pearson chi-squared test was used.

Results: Of 2,251 patients, 214 (9.5\%) had root dilaceration. Root dilacerations were determined in 276 (4.3\%) of 6386 teeth belong to 2251 patients. Anomalies were found in $9.8 \%$ of males compared with $9.3 \%$ of females. However, this difference was not statistically significant $(\mathrm{p}>0.05)$. Root dilacerations were similarly distributed between maxilla and mandible. The most frequently root dilacerated teeth were found to be mandibular third molars (12.8\%), followed by maxillary third molars (7.4\%), maxillary first molars $(6.7 \%)$. Root dilaceration was not detected in maxillary central incisors, mandibular central incisors and mandibular lateral incisors.

Conclusion: According to our results, root dilaceration is an uncommon developmental anomaly which mostly occurs in the posterior teeth that is not also possible to have trauma. The radiographic diagnosis of this anomaly is important before surgery and orthodontics treatment, but firstly endodontic treatment.
\end{abstract}

Key words: Prevalence, root dilaceration, dental radiography, Turkish. 


\section{Introduction}

Dilaceration is a deviation or bend in the linear relationship of a crown of a tooth to its root; it is an angulation or sharp curve in the root or the crown of a developed tooth of $90^{\circ}$ or more (1). There are 2 possible cause of dilaceration. The most widely accepted cause is mechanical trauma to the primary predecessor tooth, which results in dilaceration of the developing permanent tooth (2-4). An idiopathic developmental disturbances is proposed as another possible cause in cases that have no clear evidence of traumatic injury $(5,6)$.

Although dilaceration of a crown can be visually observed in an intraoral inspection, radiographic examination is required to diagnose a dilaceration of a tooth root (7). The criteria for recognizing root dilaceration vary in the literature. According to some authors $(1,8)$, a tooth is considered to have a dilaceration toward the mesial or distal direction if there is a 90-degree angle or greater along the axis of the tooth or root, whereas others defined dilaceration as a deviation from the normal axis of the tooth of 20 degrees or more in the apical part of the root (9).

When we reviewed the literature using the PubMed Database (National Library of Medicine), most publications concerning dilacerations were case reports. We found only 5 articles regarding of prevalence of dilaceration (1, 8-11). Because of the insufficient epidemiologic data, there is little information about the true prevalence of this malformation. Additionally, there was not any prevalence result with respect to root dilaceration in Turkish dental patients. The aim of this study was to determine the prevalence of root dilaceration in a Turkish dental patient population with respect to sexes, jaws, and dental localization, to determine the relation between sex and this anomaly.

\section{Materials and Methods}

We designed a descriptive study composed of periapical radiography of 2,405 patients who presented to our Maxillofacial Surgery, Oral Diagnosis and Radiology, and Endodontic Services of Dentistry Faculty, Ataturk University, in the city of Erzurum, located in the northeast part of Turkey between January 1996 and January 2008. All data (age and sex) was obtained from the files. Exclusion criteria included patients who were less than 18 years of age at the time of radiographic examination, records with poor quality radiographs and record with radiographs of only primary teeth. The final sample included 2,251 patients $(1,124$ females and 1,127 males, mean age; 37.25 years from 15 to 65 years).

Diagnosing criteria of root dilaceration: A tooth was recognized as having mesial or distal root dilaceration if there was deviation of $90^{\circ}$ or more from the normal long axis of the tooth. Orofacial direction of the dilaceration was determined by evaluating the bull's eye appearance of the root, which results from the root deviation of $90^{\circ}$ or more $(1,7,8)$. The deviation was assigned to either apical, middle, or the coronal third of the root. In multirooted teeth, a tooth was recognized as having the dilaceration of the root if at least one root showed dilaceration. In calculating the prevalence of dilaceration, the multirooted teeth having one or more dilacerated roots were counted as one case of dilaceration of the root.

To minimize variability in the present study, examinations were carried out jointly by the first two authors of the article (a researcher from Department of Oral Diagnosis and Radiology and a researcher from Department of Endodontic) over approximately one month. Throughout the study, in case of disagreement between the first two authors as to the outcome of an examination, the third author (an associated professor Department of Oral Diagnosis and Radiology) was consulted. Cases in which there was no consensus were excluded from the study.

The variables were analyzed using the Statistical Package for Social Sciences (SPSS 11.5; Chicago, IL, USA) Program. The frequencies of anomalies which are detected are calculated with respect to sexes, jaws, and dental localization. The Pearson chi-squared test was used to determine potential differences in the distribution of dental anomalies when stratified by sex. A p value of $<0.05$ was considered statistically significant.

\section{Results}

Of 2,251 patients, $276(4.3 \%)$ root dilacerations were determined in $214(9.5 \%)$ patients. Anomalies were found in $9.8 \%(\mathrm{n}=110)$ of females compared with $9.3 \%(\mathrm{n}=104)$ of males. However, this difference was not statistically significant $(\mathrm{p}=0.7)$.

Root dilacerations were similarly distributed between maxilla and mandible. The most frequently root dilacerated teeth were found to be mandibular third molars $(12.8 \%)$, followed by maxillary third molars (7.4\%), maxillary first molars (6.7\%), mandibular second molars (5.5\%), maxillary second molars (5.4\%) and mandibular first molars (5.3\%). Root dilaceration was not detected in maxillary central incisors, mandibular central incisors and mandibular lateral incisors (Table 1). The figure 1 shows examples of root dilacerations belonging different tooth types on formed periapical radiography.

\section{Discussion}

Radiographic examination is required to diagnose a dilaceration of a tooth root (7). However, panoramic radiography alone is not the method of choice for the diagnosis of root dilacerations, which can occur in the buccal/labial or palatal/lingual directions (11). Rohlin et al. (12) mentioned that periapical radiographies were superior to panoramic radiographies in detecting the lesions on periapical area. On the other hand, it was reported that panoramic radiographies are not useful tool 
Table 1. Distribution of the prevalence of root dilaceration amongst different tooth types.

\begin{tabular}{|c|c|c|c|}
\hline Tooth & $\begin{array}{c}\text { Number of } \\
\text { examined teeth }\end{array}$ & $\begin{array}{l}\text { Number of the } \\
\text { dilaceration }\end{array}$ & $\begin{array}{l}\text { Percentage of root } \\
\text { dilaceration }(\%)\end{array}$ \\
\hline Maxillary & 3,518 & 151 & 4.3 \\
\hline Central incisor & 378 & 0 & 0.0 \\
\hline Lateral incisor & 334 & 11 & 3.3 \\
\hline Canine & 351 & 4 & 1.1 \\
\hline First premolar & 468 & 15 & 3.2 \\
\hline Second premolar & 433 & 22 & 5.1 \\
\hline First molar & 571 & 38 & 6.7 \\
\hline Second molar & 579 & 31 & 5.4 \\
\hline Third molar & 404 & 30 & 7.4 \\
\hline Mandibular & 2,868 & 125 & 4.4 \\
\hline Central incisors & 282 & 0 & 0.0 \\
\hline Lateral incisor & 291 & 0 & 0.0 \\
\hline Canine & 227 & 3 & 1.3 \\
\hline First premolar & 404 & 14 & 4.6 \\
\hline Second premolar & 394 & 17 & 4.3 \\
\hline First molar & 527 & 28 & 5.3 \\
\hline Second molar & 438 & 24 & 5.5 \\
\hline Third molar & 305 & 39 & 12.8 \\
\hline Totally & 6,386 & 276 & 4.3 \\
\hline
\end{tabular}
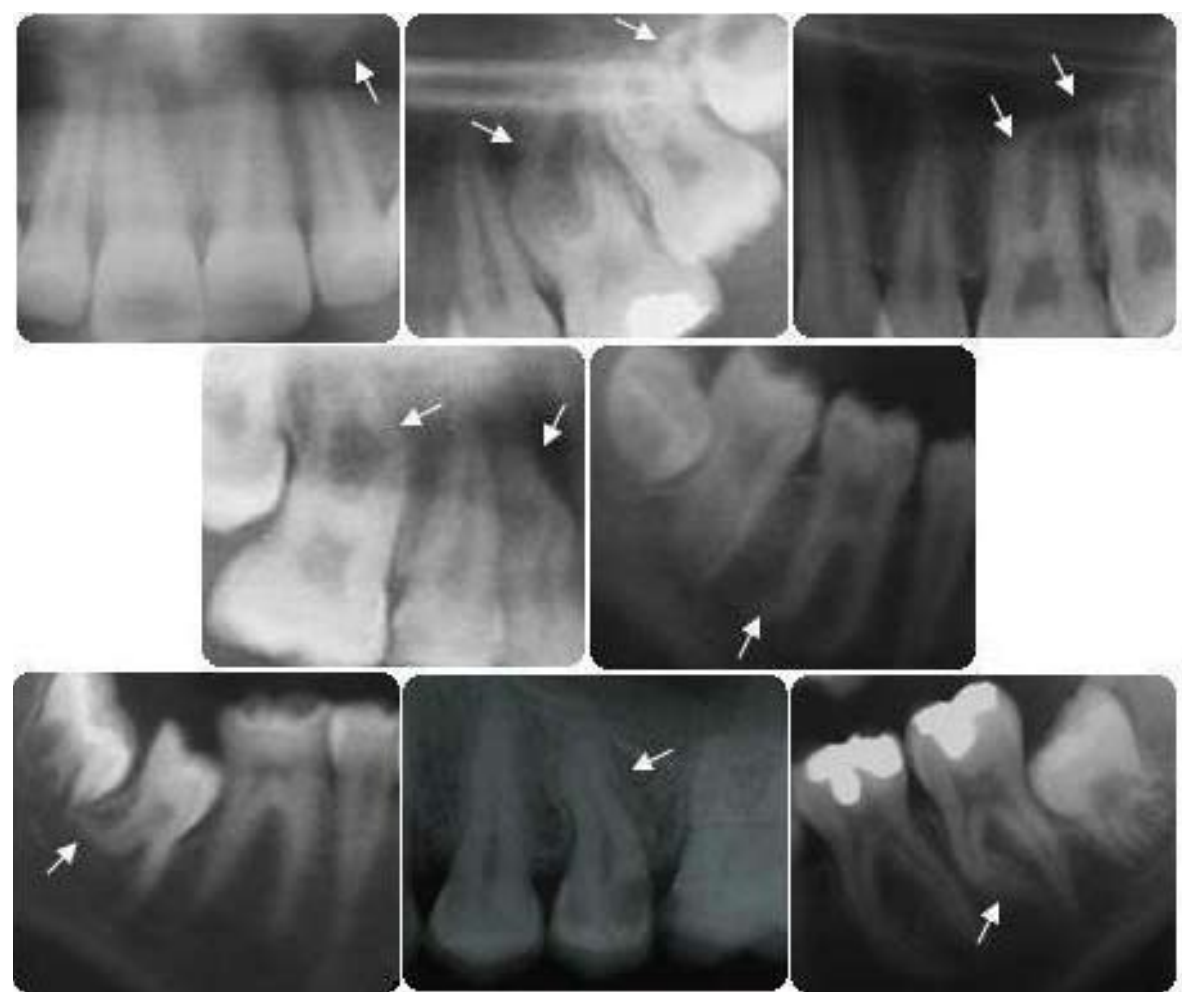

Fig. 1. Examples of root dilacerations belonging different tooth types on formed periapical radiographies. 
as periapical radiographies in an epidemiological studies of oral health (13). So, this study contains the detection of periapical radiographies which had taken from different regions for different dental problems. While Hamasha et al. (1) found out the prevalence of root dilacerations $17.0 \%$ by using periapical radiographies, Ezoddini et al. (10) and Thongudomporn et al. (11) reported the prevalence of $15.0 \%$ and $1.8 \%$ respectively by using panoramic radiographies.

Chohayeb (9) has reported that the prevalence of dilaceration in maxillary lateral incisors is $98.0 \%$; it is highly questionable whether $98.0 \%$ of teeth can be classified as having a large enough deviation to be classified as a dilaceration. The $3.3 \%$ prevalence of dilacerations of maxillary lateral incisors that we determined in this study was inconsistent with this finding. On the other hand, our results show that the prevalence is higher in posterior regions consistent with Hamasha et al. (1) and Malcić et al. (8) while root dilacerations have shown similar distributions in both two jaws in our study, it is reported that the prevalence is higher in the maxilla (8), although one other noted that two thirds of the dilacerations in their study were in the mandible (1). In the present study, found no difference between the incidence in males and females in line with some reports $(1,11)$ in the literature. However, Ezoddini et al. (10) reported that the prevalence of root dilacerations was significantly higher in males than females.

There are conflicting descriptions about the etiology of root dilacerations in the literature. Some reports $(2,3,14)$ support the role of trauma on the root dilacerations of anterior teeth. However, Andreasen et al. (5) mentioned that the major etiologic factor for dilacerations was ectopic development of tooth bud. Similarly, Chadwick et al. (6) reported dilacerated mandibular incisor that had not a trauma history but had a histological appearance consistent with trauma. Some researchers $(1,8)$ support this view because most dilacerated teeth are found in posterior teeth, and these are not prone to direct trauma similar to our results.

The success of root canal treatment depends largely on complete biomechanical debridement of the canals and the elimination of microorganisms from the root canal system. In dilacerated teeth, it is often difficult to explore and negotiate the root canals, especially if the state of the pulp has caused apposition and/or resorption of the canal wall. Therefore, when an endodontic file is introduced into the root canal during treatment, it might be blocked by such irregularities (15). Another problem in endodontic treatment of these cases is the inability to continuously follow the root canal curvature, and this might result in blocking of the canal, ledging, apical cavitation such as transportation and/or zipping, perforation, and instrument breakage (6). Therefore the diagnosis of root dilacerations before endodontic treatment has a critical importance in either preventing complications during treatment or ensuring a good treatment result.

Dilacerated root may be easily fractured during the surgical tooth extraction $(6,11)$.

Moreover, it was also reported that availability of the dilacerations in the orthodontic treatment of the teeth may make this process complicated (16).

\section{Conclusion}

This research was the first study to examine the frequency of the Turkish population of root dilaceration and one of the rare studies in the literature. Our study shows that this anomaly is distributed similarly in both jaws regardless of sex. Additionally, the dilacerations are clearly more common in the posterior teeth which are less related to trauma than the anterior teeth.

\section{References}

1. Hamasha AA, Al-Khateeb T, Darwazeh A. Prevalence of dilaceration in Jordanian adults. Int Endod J. 2002;35:910-2.

2. von Gool AV. Injury to the permanent tooth germ after trauma to the deciduous predecessor. Oral Surg Oral Med Oral Pathol. 1973;35:2-12.

3. Maragakis MG. Crown dilaceration of permanent incisors following trauma to their primary predecessors. J Clin Pediatr Dent. 1995;20:49-52.

4. Hervas A, Forner L, Llena MC. Dental manifestations of systemic disease. Med Oral. 2000;5:109-17.

5. Andreasen JO, Sundström B, Ravn JJ. The effect of traumatic injuries to primary teeth on their permanent successors. I. A clinical and histologic study of 117 injured permanent teeth. Scand J Dent Res. 1971;79:219-83.

6. Chadwick SM, Millett D. Dilaceration of a permanent mandibular incisor. A case report. Br J Orthod. 1995;22:279-81.

7. Jafarzadeh H, Abbott PV. Dilaceration: review of an endodontic challenge. J Endod. 2007;33:1025-30.

8. Malcić A, Jukić S, Brzović V, Miletić I, Pelivan I, Anić I. Prevalence of root dilaceration in adult dental patients in Croatia. Oral Surg Oral Med Oral Pathol Oral Radiol Endod. 2006;102:104-9.

9. Chohayeb AA. Dilaceration of permanent upper lateral incisors: frequency, direction, and endodontic treatment implications. Oral Surg Oral Med Oral Pathol. 1983;55:519-20.

10. Ezoddini AF, Sheikhha MH, Ahmadi H. Prevalence of dental developmental anomalies: a radiographic study. Community Dent Health. 2007;24:140-4.

11. Thongudomporn U, Freer TJ. Prevalence of dental anomalies in orthodontic patients. Aust Dent J. 1998;43:395-8.

12. Rohlin M, Kullendorff B, Ahlqwist M, Henrikson CO, Hollender L, Stenström B. Comparison between panoramic and periapical radiography in the diagnosis of periapical bone lesions. Dentomaxillofac Radiol. 1989;18:151-5.

13. Ahlqwist M, Halling A, Hollender L. Rotational panoramic radiography in epidemiological studies of dental health. Comparison between panoramic radiographs and intraoral full mouth surveys. Swed Dent J. 1986;10:73-84.

14. Diab M, elBadrawy HE. Intrusion injuries of primary incisors. Part III: Effects on the permanent successors. Quintessence Int. 2000;31:377-84.

15. Lin L, Dowden WE, Langeland K. Bilateral dilaceration. J Endod. 1982;8:85-7.

16. Tanaka E, Hasegawa T, Hanaoka K, Yoneno K, Matsumoto E, Dalla-Bona D, et al. Severe crowding and a dilacerated maxillary central incisor in an adolescent. Angle Orthod. 2006;76:510-8. 